\title{
Versorgung von hämodynamisch stabilen, mechanisch instabilen Verletzungen einschließlich Sakroiliakalgelenk
}

\author{
Jochen Pressmar, Lothar Kinzl, Michael Robindra Sarkar
}

\section{Zusammenfassung}

Beckenfrakturen sind meist Folge einer massiven Gewalteinwirkung, die häufig über zusätzliche Weichteilschäden und Begleitverletzungen zu kreislaufinstabilen Situationen führen. Dies verhindert nicht selten die wünschenswerte frühe endgültige Stabilisierung der Fraktur, weshalb Fixateur externe oder Beckenzwinge zum Einsatz kommen. Die definitive operative Stabilisierung sollte möglichst innerhalb der ersten zwei Wochen nach dem Unfall, aber nur bei stabilen Kreislaufverhältnissen erfolgen. Bis dahin muss Klarheit über das Verletzungsmuster, insbesondere die Klassifikation und damit Stabilität der Fraktur, bestehen: Stabile Frakturen (Typ A) stellen in der Regel keine Op-Indikation dar, rotationsinstabile Verletzungen des vorderen Beckenrings mit intakten dorsalen sakroiliakalen Bändern (Typ B) sind hingegen meist operationsbedürftig. Gleiches gilt bei Typ-C-Verletzungen. Bei kompletter Unterbrechung des hinteren Beckenrings ist auch eine Vertikalverschiebung der Fragmente möglich (Translationsinstabilität), die zusätzlich einer dorsalen Stabilisierung bedarf. Der operative Zugang erfolgt in der Regel über eines oder mehrere der drei Fenster des ilioinguinalen Zugangs nach
Letournel. Hierüber können sowohl Symphysensprengungen und Frakturen der vorderen Scham- und Sitzbeinäste versorgt werden (2. Fenster), als auch Frakturen des Os ilium oder der Iliosakralfuge (1. Fenster).

Zur Versorgung der Symphyse werden schmale 4-Loch-DC-Platten von kranial angelegt, Scham- und Sitzbeinfrakturen werden ebenfalls über eine Plattenosteosynthese oder alternativ über eine „Kriechschraube“ versorgt. Bei Frakturen des Os ilium oder Verletzung der Iliosakralfuge kommen Rekonstruktionsplatten resp. kurze angebogene 3-Loch-DC-Platten zur Anwendung. Neben einer exakten Reposition ist auf die Platzierung der Schrauben im Sakrum zu achten (nur eine Schraube im Bereich der Massa lateralis wegen des Verletzungsrisikos einer Nervenwurzel von L5). Am Os ilium soll die Platzierung fugennah geschehen, da nach lateral das Darmbein rasch dünn wird und keine stabile Schraubenverankerung zulässt. In speziellen Situationen am hinteren Beckenring kommen bilaterale Abstützverfahren mit Platten oder internen Fixateuren zum Einsatz, die auch die unteren lumbalen Wirbel mit einbeziehen können. Kindliche Frakturen sowie Altersfrakturen stellen Sondersituationen dar, die ein individualisiertes Vorgehen notwendig machen.

\section{Einleitung}

Das knöcherne Becken stellt den stabilsten Anteil des menschlichen Skeletts dar. Frakturen treten somit im Allgemeinen erst nach massiver Gewalteinwirkung

OP-JOURNAL 2003; 19: 118-126

(C) Georg Thieme Verlag Stuttgart - New York auf. Dies hat zur Folge, dass es sich meist um polytraumatisierte, z.T. kreislaufinstabile Patienten handelt. Vor der definitiven operativen Versorgung von Beckenfrakturen steht grundsätzlich die Stabilisierung der Vitalfunktionen. Hierzu können neben intensivmedizinischen Interventionen in der ersten Phase, auch operative Maßnahmen (z.B. Laparotomie bei intraabdominellen Blutungen, Tampona- de des Becken bei Blutungen venöser pelviner Plexus oder Reposition mit vorläufiger Stabilisierung der Beckenfraktur mittels Fixateur externe oder Beckenzwinge) oder interventionelle Verfahren (Embolisation) indiziert sein.

Während in den 70er-Jahren auch nach instabilen Verletzungen die konservative Therapie der Standard war, und der Anspruch bei operativer Versorgung von Beckenfrakturen sich auf die Stabilisierung der Fraktur konzentrierte, wird heute bei translations- (Typ C) und rotationsinstabilen (Typ B) Verletzungen Wert auf eine anatomische korrekte Rekonstruktion gelegt. Grund hierfür sind die z.T. erheblichen Beschwerdezustände bei Störungen der biomechanischen Kraftweiterleitung von der Wirbelsäule auf die untere Extremität.

Dass dies nicht immer einfach ist, erklärt sich aus der komplexen Anatomie des Beckens, der Nachbarschaft zu relevanten Organstrukturen und nicht zuletzt den Begleitverletzungen dieser Organe und des Weichteilmantels. Geklärt werden müssen außerdem die Frage nach dem geeignetsten Repositions- und Stabilisierungsverfahren, den dafür notwendigen Zugängen und dem Op-Zeitpunkt. Daraus ergibt sich auch die Lagerung auf dem Op-Tisch.

\section{Hauptteil}

Es werden Frakturen des Beckens im engeren Sinne von Frakturen des Azetabulums und des Sakrums unterschieden. Letztere werden hier nur ansatzweise beschrieben, da sich ihnen andere Kapitel dieses Heftes widmen.

Ziel der Versorgung von Beckenfrakturen ist eine möglichst korrekte Wiederherstellung der Anatomie. Da hierzu nicht selten ausgedehnte Operationsverfahren zur Anwendung kommen, ist ein kardiopulmonal stabiler Patient Voraussetzung. Aufgrund der meist vorhandenen Be- 
gleitverletzungen ist eine definitive Versorgung des Patienten selten vor dem 3. Tag möglich.

\section{Entscheidungsfindung}

Grundlegend für die Entscheidungsfindung ist neben der Kenntnis des Kreislaufzustandes und der Begleitverletzungen des Patienten eine exakte Analyse und Klassifikation der Verletzung basierend auf einer adäquaten bildgebenden Diagnostik.

Für die Entscheidungsfindung ist die Computertomographie (CT) nicht mehr wegzudenken. Dennoch kann mittels konventioneller Röntgendiagnostik der Großteil der Verletzungen korrekt diagnostiziert werden. [8] Die a.p.-Aufnahme muss hierbei durch Schrägaufnahmen (Inlet, Outlet, Ala, Obturator) ergänzt werden.

\section{Verletzungen der Symphyse}

Beurteilt werden müssen die Weite und ein möglicher Höhenversatz der Sym-
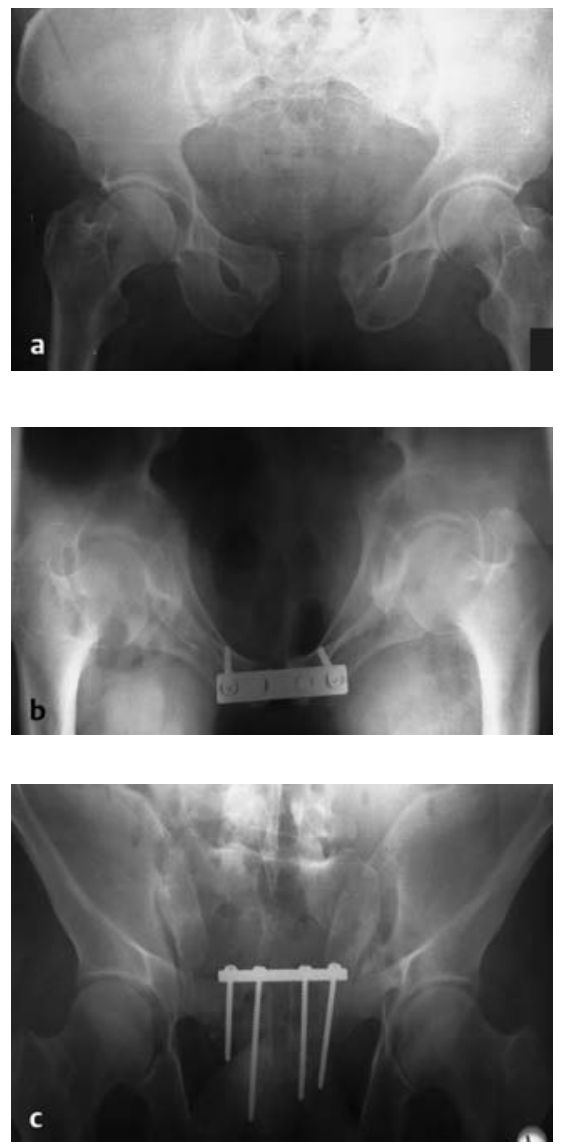

Abb.1 Open-book-Verletzung (B1, a) und Versorgung in typischer Weise mit 4-Loch-Symphysenplatte (b, c, Inlet-/Outlet-Projektion). physe. Die normale Weite der Symphyse beim Erwachsenen liegt bei ca. $10 \mathrm{~mm}$. Ein Höhenversatz ist in der a.p.-Aufnahme schwer zu beurteilen, da die Symphyse nicht orthogonal getroffen wird, so dass in Zweifelsfällen die In- und/ oder Outlet-Aufnahme herangezogen werden muss (Abb.1)

\section{Transpubische Verletzungen}

Sie entstehen meist durch geringe oder direkte Gewalteinwirkung und stellen sich als Frakturen einzelner Schamoder Sitzbeinäste dar. Bei Außenrota-
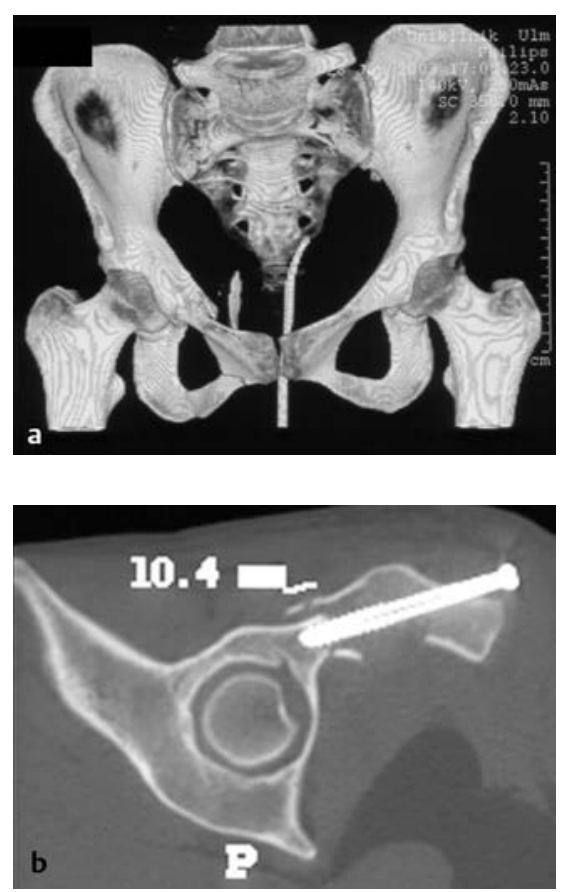

tionsverletzungen besteht häufig eine Diastase, bei Innenrotationsverletzungen eine Überlappung der Fragmente. Bei der sog. „tilt fracture“ oder dem „pubic spike“ kommt es zu Verkippungen von Fragmenten mit der Gefahr der Blasenperforation (Abb.2). Auch hier können Inlet-/Outlet-Aufnahme bei der Beurteilung der Lage dieser Fragmente hilfreich sein.

\section{Transiliakale Verletzungen}

Diese Frakturen sind übersichtsradiographisch aufgrund der unterschiedlichen Knochendichten am Becken und der Überlagerungsphänomene durch Darminhalt, aber auch der Knochenflächen selbst meist schwer zu beurteilen. Oft weisen diese Verletzungen multiple und variable Frakturlinien auf. Eine Alaoder Obturatoraufnahme, ggf. eine CT, kann zur Interpretation und Op-Planung notwendig werden.

\section{Transiliosakrale Verletzung}

Durch den schrägen Verlauf der ISG-Fuge ist die radiologische Diagnose von Verletzungen dieses Gelenks schwierig, eine Beurteilung im Seitenvergleich dabei fast immer hilfreich. Beurteilt wird die Weite des Gelenkspaltes, die Parallelität der iliakalen und sakralen Gelenkkontur sowie ein Höhenversatz. Besteht ein Höhenversatz oder ist das SI-Gelenk über $10 \mathrm{~mm}$ erweitert, muss von einer Translationsverletzung (Typ C) ausgegangen werden (Abb. 3). Ist das SI-Gelenk ledig-
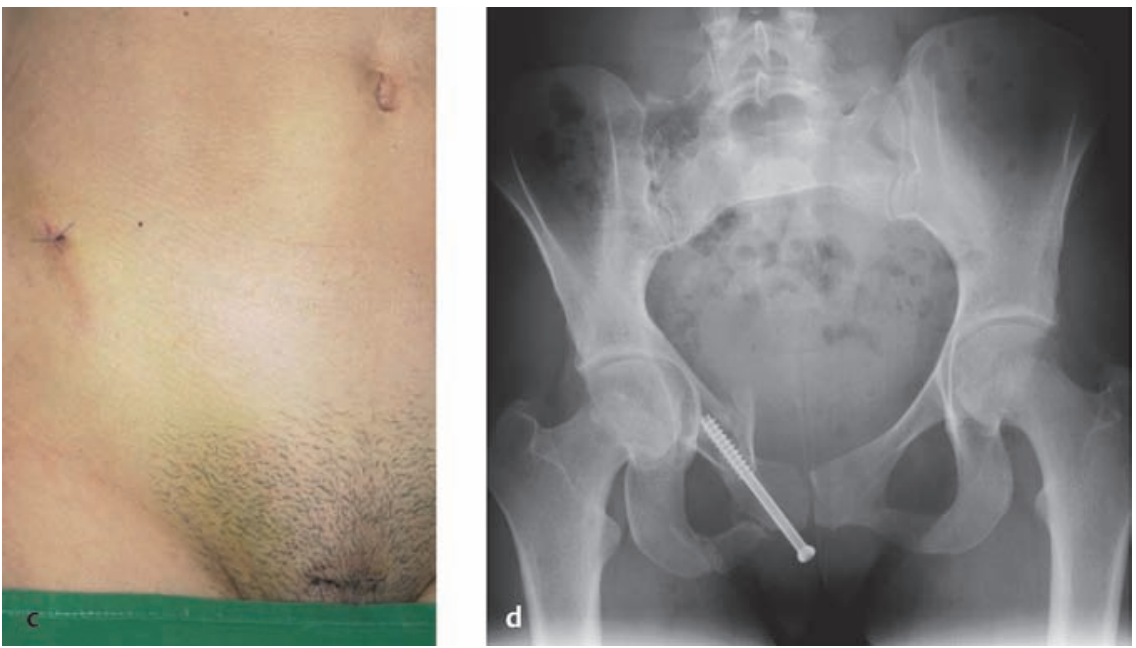

Abb.2 Laterale Kompressionsverletzung (B2) mit ausgebrochenem Schambeinfragment („pubic spine“, a). Da keine Blasenperforation vorlag, wurde das Fragment belassen und eine navigierte Verschraubung über Stichinzision durchgeführt (b, c). Vor Metallentfernung ist das Fragment bereits partiell resorbiert (d). 
lich ventral aufgeklappt, handelt es sich aller Wahrscheinlichkeit nach um eine Rotationsverletzung (Typ B). Bei Innenrotationsverletzungen kann es zur Impressionsfraktur der Massa lateralis des Sakrums kommen, mit z.T. nur minimal verändertem SI-Gelenk. Auch hier helfen im Zweifelsfall In- und Outlet-Aufnahmen oder ggf. eine CT.

\section{Extrapelvine Befunde}

Frakturen der Querfortsätze der LWS, und hier insbesondere des 5. LWK, weisen auf das Vorliegen einer Translationsverletzung vom Typ $\mathrm{C}$ hin.

\section{Indikation}

Im Wesentlichen legt die Frage der verbliebenen Stabilität die OP-Indikation fest:

- Stabile Frakturen (Typ A) wie Abrissfrakturen, Beckenrandbrüche oder nicht dislozierte vordere Beckenringbrüche stellen in der Regel keine OPIndikation dar und werden der konservativen Therapie zugeführt. Ausnahmen sind Frakturen, die ungeachtet ihrer Stabilität aufgrund der Stellung der Fragmente, Probleme bereiten (Weichteilschädigung, Schmerzen oder Einschränkungen der Mobilisation,...) oder Abrissfrakturen bei Leistungssportlern.

- Partiell instabile Verletzungen (Typ B) zeichnen sich durch intakte dorsale sakroiliakale Bänder aus. Hierdurch wird zwar eine vertikale Verschiebung der Fragmente verhindert, aufgrund der Kontinuitätsunterbrechung des vorderen Beckenrings können sie sich jedoch gegeneinander um eine vertikale Achse drehen, weshalb diese Situation als rotationsinstabil bezeichnet wird. Hier besteht in der Regel eine OP-Indikation. Häufig handelt es sich hierbei nicht um eine Fraktur des vorderen Beckenrings, sondern um eine Sprengung der Symphyse als schwächstem Glied des Beckengürtels. Es kommt zum ventralen Aufklappen des Beckens, was als „open-book-Verletzung“ bezeichnet wird. Besteht eine Diastase von mehr als $2,5 \mathrm{~cm}$ oder eine klinische Instabilität, besteht die Indikation zur Operation. Ausnahme kann bei Typ-B-Verletzungen eine wenig dislozierte Innenrotationsverletzung sein, wenn es zur stabilen Einstauchung der Fragmente kommt. Somit ist bei den B-Verletzungen im Normalfall die ventrale Stabilisierung ausreichend, wofür verschiedene Verfahren (Platte,
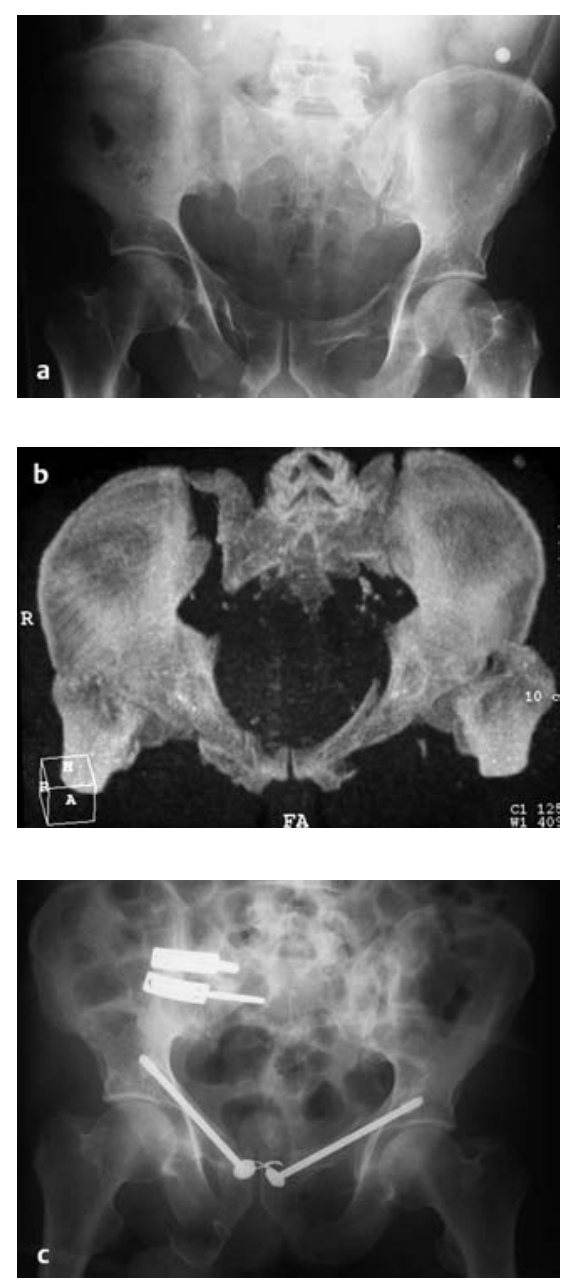

Abb. 3 Translationsverletzung rechts und laterale Sakrumkompression links (Typ C2), Stabilisierung mit ISG-Platten und Kriechschrauben in beiden Schambeinästen (c).

Fixateur externe, Kriechschraube) in Betracht kommen (Abb.1 u.2).

- Bei Verletzungen mit Zerreißung der dorsalen sakroiliakalen Bandstrukturen (Typ C) kommt es zur kompletten Instabilität, bei der sich die Fragmente gegeneinander drehen und zusätzlich vertikal verschieben können (Abb.3,4 u.6). Bei diesen sog. Translationsinstabilitäten besteht immer eine OP-Indikation. Eine ausschließlich ventrale Stabilisierung ist in diesen Fällen nicht ausreichend, um die dorsalen Strukturen ebenfalls in anatomischer Position zu halten, deshalb ist eine Stabilisierung am dorsalen Beckenring zusätzlich vonnöten. Hier wird je nach Lokalisation der Frakturlinien und dem Ausmaß des Gewebetraumas eines der im Folgenden genannten Verfahrens ausgewählt.
Während A-Verletzungen als stabil gelten und in der Regel konservativ behandelt werden, ist bei B-Verletzungen eine ventrale Osteosynthese und bei C-Verletzungen zusätzlich eine dorsale Stabilisierung indiziert.

\section{OP-Zeitpunkt}

Grundsätzlich gilt für instabile Beckenringverletzungen, dass eine frühe Stabilisierung dem Patienten Schmerzen erspart und die Frakturheilung positiv beeinflusst. Berücksichtigt werden müssen jedoch die Begleitverletzungen und der Kreislaufzustand des Patienten. Sollten diese die sofortige Versorgung nicht erlauben, muss auf eine temporäre Stabilisation mittels Beckenzwinge oder Fixateur externe ausgewichen werden. Eine definitive Versorgung sollte möglichst innerhalb der ersten Woche, jedoch spätestens nach 14 Tagen erfolgen, da aufgrund der raschen Kallusbildung die Reposition anschließend deutlich erschwert wird.

Die definitive operative Stabilisierung sollte möglichst innerhalb der ersten zwei Wochen nach dem Unfall, aber nur bei stabilen Kreislaufverhältnissen erfolgen.

\section{OP-Verfahren}

Bei jeder Osteosynthese am Beckenring ist vor dem Abdecken zu prüfen, ob die intraoperative Durchleuchtung in allen notwendigen Einstellungen einschließlich der Schrägprojektionen unbeeinträchtigt möglich ist. Die Lagerung des Patienten richtet sich nach dem geplanten Vorgehen.

\section{Symphysensprengung}

Die Versorgung einer Symphysensprengung geschieht in Rückenlage.

Nach Freilegung der Symphyse über einen horizontalen suprapubischen Schnitt („Pfannenstielschnitt“) oder im Anschluss an eine mediane Laparotomie erfolgt die Reposition mittels einer Repositionszange (Abb. $\mathbf{8}$ ), die beidseits im Corpus ossis ischii am Oberrand des Foramen obturatum eingesetzt wird. Die Reposition wird bei weit klaffender Symphyse erleichtert, wenn vor der sterilen Abdeckung die beiden Knie mit einer kräftigen elastischen Binde gegeneinander gewickelt werden.

Die Stabilisierung erfolgt typischerweise durch Einbringen einer 4-Loch-Platte 

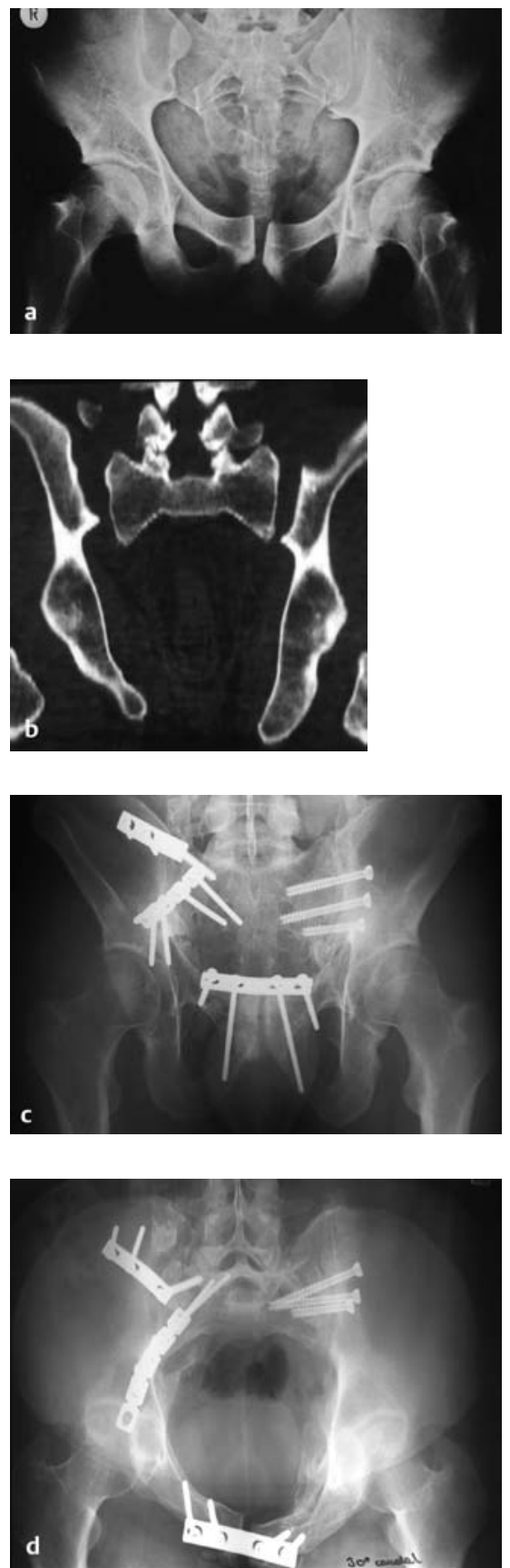

Abb. 4 C2-Verletzung mit deutlich erkennbarer Translation rechts und Rotationsinstabilität ohne Vertikalversatz links (b). Stabilisierung mit ISG-Platten rechts, navigierter Verschraubung links und Symphysenplatte (c, d: Ausheilungsbilder in Inlet- und OutletProjektion).

(4,5-mm-DCP schmal). Für die Stabilität der Versorgung ist hierbei wichtig, dass die Platte dorsal des Tuberculum pubicum, hinter dem Ansatz des M. rectus abdominis platziert wird. Die Enden der Platte sollten um ca. $10-15^{\circ}$ nach unten gebogen werden, um die Schraubenrichtung zu optimieren. Die medialen Schrauben werden parallel zur Symphyse einge- bracht, die lateralen sollen mit ihrer Spitze auf die Spitze der medialen Schrauben zielen (Abb.1 u.4). Beim Bohrvorgang wird mit dem Zeigefinger die innere Kortikalis des Corpus ossis pubis und der Discus interpubicus palpiert, um die Bohrrichtung kontrollieren zu können. Durch dieses Vorgehen lassen sich Schraubenlängen von 50 bis $70 \mathrm{~mm}$ erzielen.

Um Wundheilungsstörungen u.v.a. die Ausbildung einer Hernie zu vermeiden, ist ein exakter schichtweiser Wundverschluss erforderlich.

Die Implantatentfernung ist für 6 bis 12 Monate nach Verletzung vorzusehen, da andernfalls die Platte bricht oder sich die Schrauben unter der Wechselbelastung beim Gehen lockern.

\section{Fraktur des vorderen Beckenrings}

Sollten Anteile des ventralen Beckenrings lateral der Symphyse frakturiert sein, wird über einen ilioinguinalen Zugang oder über einen „Pfannenstiel“-Zugang eine längere Rekonstruktionsplatte unter die in der Leiste verlaufenden Gefäße und Nerven platziert (Abb.5). Hierzu ist eine sorgfältige Präparation mit Anschlingen dieser Strukturen erforderlich. Aufgrund der möglichen Komplikation einer Gefäß-/Nervenverletzung oder auch der Ausbildung einer Lymphfistel kann alternativ eine „Kriechschraube“ in den oberen Schambeinast eingebracht werden, die als innere Schienung dient (Abb.2u.3).

Insbesondere bei beidseitigen ventralen Beckenringfrakturen kann die für eine Plattenosteosynthese notwendige weite Freilegung durch die Anlage eines supraazetabulären Fixateurs vermieden werden. Dem Nachteil einer möglichen Infektion der Pindurchtrittstellen und des schlechteren Patientenkomforts steht ein wesentlich geringeres OP-Trauma gegenüber (Abb.6).

Frakturen des Os ilium und Sprengung des sakroiliakalen Gelenks

Die Stabilisierung der dorsalen Anteile des Beckenrings erfolgt abgesehen von bilateralen ISG-Sprengungen und Sakrumfrakturen (hier sind Bauchlage und dorsaler Zugang empfehlenswert) in Rückenlage über einen anterolateralen Zugang. Dies entspricht dem sog. 1. Fenster des ilioinguinalen Zugangs n. Letournel (s. unten). Ein in die Massa late-
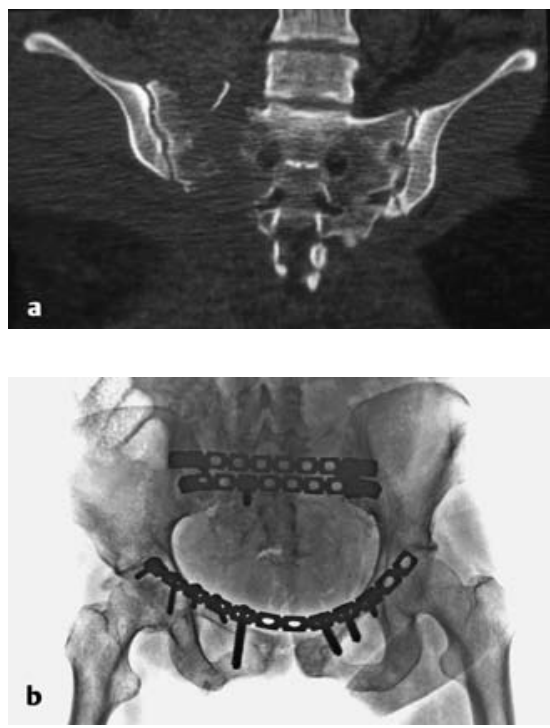

Abb.5 Extrem adipöse und unkooperative Patientin mit C2-Verletzung. Verlauf durch die Massa lateralis des Sakrums (a). Stabilisierung mit ilio-iliakalen Platten und langer Rekonstruktionsplatte für den gesamten vorderen Beckenring (b).

ralis des Sakrums eingesetzter Hohmann-Hebel hält die Weichteile nach medial. Dabei ist auf der Vorderfläche des Sakrums die hier verlaufende Wurzel des 5.lumbalen Segmentes zu schonen.

Zur Erleichterung der Reposition kann eine Schanz-Schraube mit T-Griff im Beckenkamm fixiert und als Steuerungsinstrument verwendet werden. Wichtig ist hier wie bei Frakturen des ventralen Beckenringes, das Bein der verletzten Seite mobil abzudecken, da durch Zug und Rotation die Reposition wesentlich unterstützt werden kann.

Nach Reposition wird die zerrissene Ileosakralfuge von ventral mit zwei kurzen angebogenen Platten (meist 4,5-mmDCP 3-Loch) stabilisiert. Diese Platten sollten in einem Winkel von $70-90^{\circ} \mathrm{zu}-$ einander positioniert werden, da die Schrauben dann den stabilsten Knochen entlang der Linea terminalis einerseits und dem Beckenkamm andererseits fassen [5] (Abb.4). Lediglich eine Schraube wird in die Massa lateralis des Sakrums gesetzt, da medial davon die Nervenwurzel L5 verläuft; zwei Schrauben werden in das Os ilium eingebracht.

Die Kontrolle über die Reposition und die Lage der Schrauben ist durch direkte Sicht nicht ausreichend und muss deshalb mittels Bildwandler (a.p.-, Inlet-/ 

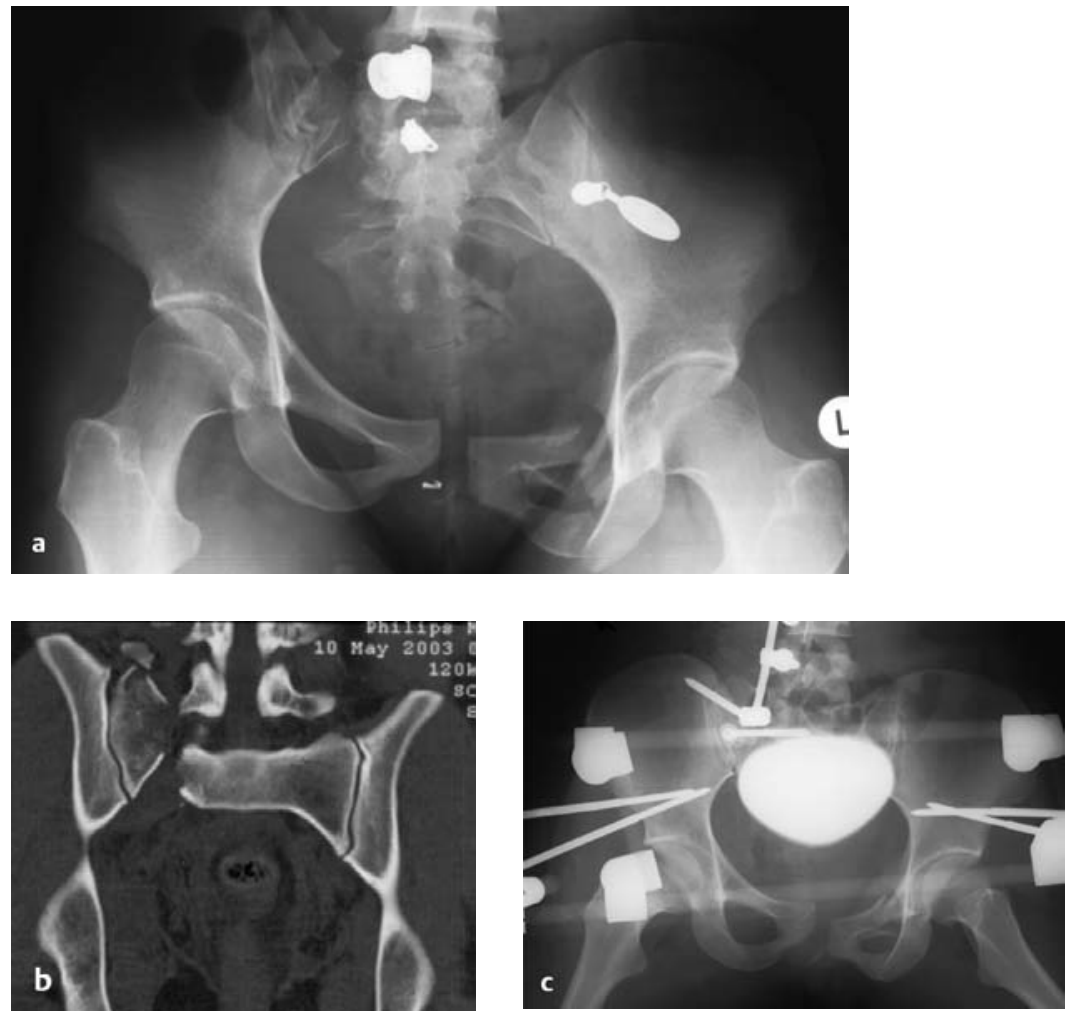

Abb.6 C1-Verletzung nach Reitunfall mit deutlichem Höhenversatz. Rekonstruktion mit vertebropelviner Abstützung von L4/5 auf das Os ilium (USS-System mit polyaxialen Schrauben) und supraazetabulärem Fixateur ventral, um eine ausgedehntere Präparation des vorderen Beckenringes zu umgehen (b).

Outlet-Projektion) erfolgen. Neuerdings bietet sich die Möglichkeit der Durchführung einer intraoperativen Computertomographie, die eine wesentlich verbesserte Beurteilung der Implantatlage gestattet.

Gefährdet ist bei diesen Osteosynthesen der Truncus lumbosacralis, der sich dem SI-Gelenk von ventral medial bis auf $1 \mathrm{~cm}$ nähern kann. Einer gewissen Gefahr ist auch der N.obturatorius ausgesetzt, der intramuskulär zwischen dem M.iliacus und psoas major nahe der Linea terminalis verläuft.

Eine alternative Methode zur ventralen Plattenosteosynthese der ISG-Fuge besteht in der Transfixation des ISG mit kanülierten Schrauben, die aber nur durchgeführt werden sollte, wenn am Os sacrum keine ausgedehnte transforaminale Trümmerzone vorliegt (Abb.4). Die Verschraubung kann in Rückenlage wie in Bauchlage durchgeführt werden. Die Technik ist anspruchsvoll und erfordert zwingend eine perfekt eingestellte Durchleuchtung in den schrägen (Inlet-/ Outlet-)Ebenen und seitlich. Neben die herkömmliche offene Verschraubung ist
- unterstützt durch rechnergestützte Navigationshilfen - das transkutane navigierte Vorgehen getreten.

Die Implantatentfernung sollte wenn überhaupt nur beim jungen Patienten nach 6 bis 12 Monaten erfolgen. Bei Verknöcherung der SI-Fuge kann, falls der Patient keine Beschwerden hat, auf die Metallentfernung verzichtet werden. Generell ist am Becken der Nutzen einer Metallentfernung gegen die aufwändige Freilegung und das Risiko einer dabei auftretenden Verletzung z. B. der Wurzel L5 abzuwägen.

Bei Luxationsfrakturen mit Beteiligung des dorsalen Os ilium oder der Massa lateralis des Sakrums kommen Osteosynthesen in Betracht, die in Bauchlage ausgeführt werden. Bei beidseitiger Sprengung der ISG-Fuge oder Zertrümmerungen des Sakrums werden dorsale ilio-iliakale Abstützungen verwendet. Dies kann mit einer durchgeschobenen Platte (Abb.5) oder auch mit einem Fixateur interne geschehen. Allerdings ist dabei darauf $z u$ achten, dass die Implantate möglichst wenig auftragen, da sonst Druckulzera und Infekte drohen. Weiter- hin muss wie bereits erwähnt bei Trümmerfrakturen des dorsalen Beckenrings, und hier besonders des Sakrums, eine Kompression der Fraktur durch Zugschrauben vermieden werden, da es sonst zur Schädigung des sakralen Plexus kommen kann. Liegt bereits durch das Trauma ein Nervenschaden vor, so wird eine Dekompression des sakralen Spinalkanals empfohlen. Platten oder Fixateure werden unter Distraktion eingesetzt. Genauere Kenntnisse über Sakrumfrakturen ermöglichen heute auch so genannte „lokale Osteosynthesen“ mit Kleinfragmentinstrumentarium direkt am Sakrum.

Schließlich spielt die Option einer sog. vertebropelvinen Abstützung eine Rolle, d.h. einer Verstrebung zwischen dem dorsalen Beckenkamm und an den kaudalen Lendenwirbeln (L4, L5) eingebrachten transpedikulären Schrauben (Abb. 6). Mit dieser Montage werden die starken dislozierenden Muskelkräfte, die die verletzte Seite des Beckens nach kranial verschieben, neutralisiert.

\section{Zugangswege}

In kaum einer anderen Körperregion sind so viele Zugangswege beschrieben wie beim Hüftgelenk und Becken. Grundsätzlich unterscheidet man limitierte von erweiterten Zugängen. Während limitierte Zugänge sich v.a. durch ihre Weichteilschonung auszeichnen, sind die erweiterten Zugänge durch ihre gute Übersicht, aber auch durch häufigere postoperative Komplikationen aufgrund des größeren Weichteilschadens charakterisiert. Topographisch können die Zugänge in anteriore, laterale und posteriore Zugänge unterschieden werden.

\section{Limitierte Zugänge}

Der posteriore Zugang nach KocherLangenbeck ist eine Kombination des dorsalen Zugangs von Langenbeck und des dorsalen Zugangs von Kocher und entspricht heute dem Standardzugang zum Azetabulum bei der Versorgung von hinteren Pfannenrandfrakturen, hinteren Pfeilerfrakturen, Querfrakturen und T-Brüchen sowie doppelten Pfeilerbrüchen, deren Hauptdislokationsrichtung nach dorsal gerichtet ist. Für die Stabilisierung extraartikulärer Beckenringfrakturen spielt dieser Zugang keine wesentliche Rolle.

Der ilioinguinale Zugang nach Letournel wurde ebenfalls zur Versorgung von Azetabulumfrakturen eingeführt. Er er- 
möglicht eine gute Übersicht über den gesamten ventralen Pfeiler und kann nach dorsal bis zur ISG-Fuge ausgedehnt werden. Er kommt bei Iliumfrakturen, transiliakalen Luxationsfrakturen sowie bei Azetabulumfrakturen, deren Hauptdislokationsrichtung nach ventral verläuft, zur Anwendung. Von ihm leiten sich zahlreiche Modifikationen und Erweiterungen $a b$.

Der Patient liegt in Rückenlage oder Seitenlage entweder auf einem Normaloder auf einem Extensionstisch. Der Hautschnitt beginnt am höchsten Punkt der Darmbeinschaufel, verläuft zur Spina iliaca anterior superior, folgt dem Leistenband nach medial und endet ca. $2 \mathrm{~cm}$ oberhalb der Symphyse.

Durch diesen Zugang können drei sog. „Fenster“ exploriert werden. Das 1.Fenster führt auf die Fossa iliaca, das 2. Fenster zusätzlich auf den ventralen Pfeiler und das 3. Fenster auf die Innenseite des vorderen Beckenrings und die Symphyse.

Zunächst wird die seitliche Bauchmuskulatur von der Crista iliaca abgelöst und der M.iliacus aus der Fossa iliaca subperiostal nach medial bis zur Linea terminalis stumpf abgedrängt. Ventral der Spina ist der N.cutaneus femoris lateralis darzustellen und falls möglich zu schonen. Nach Inzision der Aponeurose des M.obliquus externus wird der Samenstrang dargestellt und angeschlungen. Nach Ablösung der tiefen Bauchwandmuskeln vom Leistenband wird der Arcus iliopectineus durchtrennt. Anschließend wird der M. iliopsoas mit dem N. femoralis unterfahren und angeschlungen. Eine dritte Schlinge nimmt das Gefäßbündel auf, welches zur Schonung der Lymphbahnen so wenig wie möglich disseziert werden soll. Der gesamte ventrale Pfeiler und die Innenseite der Beckenschaufel sind nun frei zugänglich.

Je nach Verlauf der Fraktur ist es nicht immer erforderlich, alle dieser Fenster bis auf den Knochen zu präparieren, damit kann das Weichteiltrauma geringer gehalten werden. Andererseits kann dieser Zugang bei Bedarf auch auf die Gegenseite verlängert werden.

Der iliofemorale Zugang hat die Bedeutung für die Versorgung von vorderen Pfeilerfrakturen zugunsten des ilioinguinalen Zugangs verloren und soll hier deswegen nicht näher ausgeführt werden. Er kommt noch bei der Versorgung von Hüftkopffrakturen zur Anwendung.

\section{Erweiterte Zugänge}

Die wichtigsten erweiterten Zugänge für die Versorgung von Azetabulumfrakturen sind

- der erweiterte iliofemorale Zugang nach Judet

- der „Maryland“-Zugang nach Reinert a der laterale gerade Zugang nach Ruedi

Die erweiterten Zugänge am Becken dienen vorwiegend der Osteosynthese komplexer Gelenkfrakturen, die über einen limitierten Zugang nicht ausreichend reponiert und retiniert werden können, sowie für späte Versorgungen nach der 3 . Woche und für sekundäre Korrektureingriffe. Die Stabilisierung von frischen Beckenringverletzungen kommt dagegen in aller Regel ohne diese aufwändigen und komplikationsträchtigen Freilegungen aus.

\section{Dorsale Zugänge}

Für dorsale Stabilisierungen in Bauchlage werden längs verlaufende Inzisionen bevorzugt. Diese können median oder paramedian verlaufen, wenn die Rückfläche des Sakrums beidseitig oder einseitig erreicht werden soll. Für vertebropelvine Abstützungen bewährt sich ein schräg von paramedian auf die Spina iliaca posterior zu verlaufende Inzision. Ilio-iliakale Abstützungen werden möglichst mit zwei längs über den dorsalen Beckenkämmen verlaufenden Zugängen und durchgeschobenen Implantaten realisiert. Bei allen dorsalen Zugängen ist ein weichteilschonendes Operieren und eine spannungsfreie Bedeckung der Implantate von entscheidender Bedeutung, da es sonst unweigerlich zu Heilungsstörungen und Infekten kommt.

\section{Besondere Situationen}

\section{Kindliche Frakturen}

Beckenfrakturen im Kindesalter sind aufgrund der höheren Elastizität der Knochen-Bandverbindungen sehr selten. Der vorherrschende Verletzungstyp sind A-Frakturen. Wurde jedoch eine Beckenfraktur vom Typ B oder C diagnostiziert, so muss von einer starken Gewalteinwirkung mit der Möglichkeit konsekutiver Begleitverletzungen, insbesondere intraabdominell, ausgegangen werden. Dies ist umso wichtiger, als Kinder Blutverluste sehr lange kompensieren können. Liegt eine Beckenverletzung vor, und lässt der Unfallmechanismus eine entsprechende Gewalteinwirkung vermuten (oft Überrolltraumen), so muss wegen der Gefahr einer hämorrhagischen Dekompensation zügig das Ausmaß der Begleitverletzungen geklärt werden. Dies hat auch zur Folge, dass nach einer initialen konventionellen a. p.-Röntgenaufnahme des Beckens bei Unklarheit über das Vorliegen und Ausmaß einer Fraktur eine CT durchgeführt werden sollte, die eine exakte Beurteilung der knöchernen und Weichteilstrukturen zulässt.

Für die Beurteilung der bildgebenden Diagnostik müssen die altersabhängige Breite des Symphysenspaltes sowie die im Vergleich zum Erwachsenen breiteren IS-Fugen berücksichtigt werden. Prinzipiell wird die Indikation zur operativen Versorgung von Beckenfrakturen bei Kindern zurückhaltender gestellt, weil in dieser Altersgruppe plastische Deformationen auftreten können, die nicht instabil sind. So werden „Open-book-“ und andere Typ-B-Verletzungen beim Kind in Abhängigkeit vom Ausmaß der Dislokation konservativ oder operativ versorgt. Bei Instabilität oder erheblicher Dislokation ist aber wie auch bei Typ-C-Verletzungen die OP-Indikation gegeben. Zur Versorgung empfehlen sich je nach Alter kleinere Implantate wie die 3,5-mm-DCP, 2,7-mm-DCP oder die Drittelrohrplatte der AO, wobei jeweils 2 besetzte Schraubenlöcher pro Fragment ausreichend sind [7].

\section{Altersfrakturen}

Die Häufigkeitsverteilung von Beckenfrakturen zeigt beim männlichen Geschlecht einen Altersgipfel bei 30 Jahren und einen weiteren in der Mitte des 5.Lebensjahrzehntes, während bei Frauen ein Anstieg zwischen dem 70. und 85.Lebensjahr zu verzeichnen ist. Insgesamt steigt die Inzidenz von Altersfrakturen und hier insbesondere von osteoporosebedingten Frakturen deutlich an. So konnte in Finnland zwischen 1970 und 1991 eine Verdreifachung beobachtet werden [3]. Hierbei sind instabile Beckenfrakturen im Alter sehr selten, ihre Prognose aufgrund der deutlich eingeschränkten kardio-pulmonalen Kompensationsfähigkeit ist dafür sehr schlecht. So ist bei Typ-C-Verletzungen bei über 65-Jährigen mit einer dreifach höheren Letalität zu rechnen im Vergleich zu unter 50-Jährigen [4].

Das hat zur Konsequenz, dass einerseits instabile Frakturen sofort mechanisch stabilisiert (Fixateur externe, Beckenzwinge) und Blutungen gestillt (frühzeitige Exploration und Tamponade) werden 
müssen, aber andererseits eine definitive Osteosynthese erst nach sicherer Kreislaufstabilisierung des Patienten erfolgen darf. Es ist besonders auf eine penible Blutstillung und eine schonende Operationstechnik zu achten. Aufgrund der osteoporotisch geschwächten Knochenstruktur muss die maximal mögliche Schraubenlänge ausgenutzt werden.

Bei osteoporotischen Frakturen (inadäquates Trauma) bei denen häufig Sitz- und Schambeinast betroffen sind, muss eine Verletzung des hinteren Beckenrings ausgeschlossen werden (klinisch: tiefe Rückenschmerzen, Kompressionsschmerz bei lateraler Kompression des Beckenrings, radiologisch: Inlet-/Outlet-Aufnahme, ggf. CT). Das weitere Vorgehen ist möglichst konservativ. So werden nicht dislozierte und eingestauchte Frakturen konservativ (kurze Bettruhe und Analgetika) behandelt. Bei Beschwerdepersistenz erfolgt nach ca. einer Woche eine erneute röntgenologische Kontrolle. Bei Fällen mit sekundärer Dislokation ist in Abhängigkeit von der Symptomatik und dem Allgemeinzustand die Indikation zur Osteosynthese individuell zu stellen. Aufgrund der oft hohen Komorbidität ist die Frühmobilisation das Ziel.

\section{Das Komplextrauma}

Becken- oder Azetabulumfrakturen mit zusätzlichen peripelvinen Verletzungen des Haut-Muskel-Mantels, des Urogenitalsystems, des Darms, der großen Gefäße und/oder der großen Nervenbahnen werden als komplexe Beckenverletzungen bezeichnet.

Am häufigsten werden Verletzungen des Urogenitaltrakts beobachtet (ca. 65\%). Blasenrupturen (ca. 43\%) liegen hierbei vor Urethraverletzungen (ca. 26\%) und Verletzungen der Vagina (ca. 8\%) oder des Uterus (ca. 1-2\%). Für die Blasenoder Urethraverletzung ist Blut im Urin dabei der führende klinische Hinweis, der Anlass zu einer retrograden Urethrobzw. Zystographie gibt. Bei Verdacht auf eine Urethraverletzung muss auf einen transurethralen Katheter verzichtet, statt dessen ein suprapubischer angelegt werden. Nach Stabilisierung der Vitalfunktionen erfolgt die Versorgung durch den Urologen, hierbei sollte ggf. die primäre operative Versorgung der Beckenfraktur in gleicher Sitzung angestrebt werden. Nach primärer Reanastomosierung der Harnröhre bleiben in bis zu 50\% der Fälle Harnröhrenstrikturen bestehen. Die Rate der erektilen Dysfunktionen liegt zwischen 60 und $80 \%$. Bei sekundärer Versorgung liegen die Werte bei 90 bzw. $15-20 \%$. [5]

Bei pelvinen Darmverletzungen wird der Darm primär ausgeklemmt und abgestopft. Die Anlage eines doppelläufigen Kolostomas erfolgt nach Kontrolle der hämodynamischen Situation. Es sollte möglichst fern der Beckenwunde angelegt werden. Der abführende Schenkel wird am Ende der Operation ausgespült und bleibt in der Regel offen. Auf einen definitiven Verschluss des Abdomens sollte verzichtet werden, um nicht druckbedingten Ventilationsstörungen oder Durchblutungsstörungen des Darms Vorschub zu leisten. Bei diesen schweren Verletzungen sind regelmäßige Revisionen in kurzen Abständen erforderlich.

Beckenverletzungen mit Weichteilverletzungen lassen sich in offene und geschlossene Verletzungen einteilen (Abb. 7).

Zu den offenen Verletzungen zählen auch die Pfählungsverletzungen. Die Letalität der offenen Verletzungen wird mit bis zu 50\% angegeben. Meist ist hierbei die Todesursache eine nicht beherrschbare Blutung oder Sepsis (70\%). Das primäre Ziel bei diesen Beckenverletzungen ist neben der Blutungskontrolle die Evaluation des Verletzungsmusters. Weitere operative Schritte sollten in einer zweiten sog. „Second-look“-Operation erfolgen. Die osteosynthetische Versorgung ist bei diesen schwerst verletzten Patienten nicht nach Schema, sondern individuell nach Weichteilsituation und erreichter kardiopulmonaler Stabilität des Patienten zu planen.

$\mathrm{Zu}$ den Beckenverletzungen mit Weichteilverletzungen zählt auch das Kompartmentsyndrom des Beckens, das durch Hämatome und Schwellungen gekennzeichnet ist und zur Schädigung großer

Abb. 7 Komplextrauma mit weit aufgerissenen Weichteilen im Bereich des Dammes und der Leiste, C1-Verletzung (a). Zuverlegung nach auswärtiger Anlage einer Beckenzwinge und Bauchtuchtamponade bei instabilen Kreislaufverhältnissen (b). Wechsel auf supraazetabulären Fixateur externe (c) und perkutane ISG-Verschraubung (d), da aufgrund der Weichteilsituation keine andere Osteosynthese vertretbar war (e).
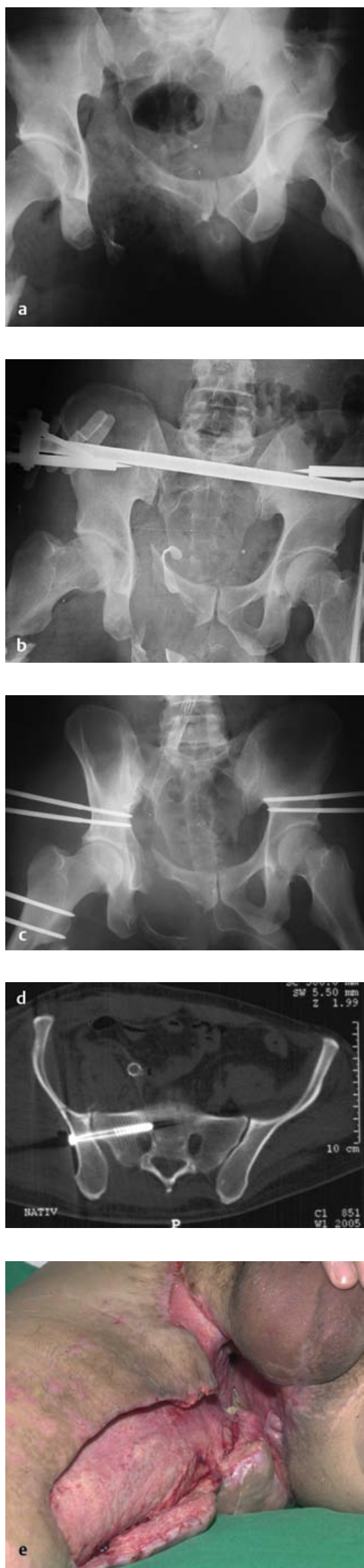
Nerven führen kann. (Nn.ischiadicus, femoralis, obturatorius). Auch Muskelnekrosen als Folge des Kompartmentsyndroms können zu systemischen Auswirkungen führen (Crush-Syndrom). Initial ist die Entlastung, sekundär die Ausräumung notwendig. Bei Verschmutzungen kann zur Wundkonditionierung und beim Kompartmentsyndrom zum spannungsfreien Wundverschluss eine Vakuumversiegelung erfolgen.

Eine besondere Weichteilschädigung, die v.a. bei Überrolltraumen durch die auftretenden Scherbewegungen zustande kommt, ist die sog. Morel-Levallé-Läsion. Hierbei löst sich das Unterhautfettgewebe von der Faszie. Die entstandene Höhle füllt sich mit Blut und Lymphflüssigkeit, so dass es auch bei geschlossenen Verletzungen häufig zu sekundären Infekten kommt.

Nervenläsionen haben für die Erstversorgung eines Komplextraumas bei fehlender Therapiemöglichkeit keine Konsequenz. Sie kommen in bis zu einem Drittel der Fälle vor.

\section{Komplikationen}

Trotz einer differenzierten Indikationsstellung zu Reposition und Stabilisierung am Beckenring sind die langfristigen Ergebnisse nicht durchgehend positiv. Viele Patienten leiden dauerhaft unter Schmerzen oder anderen Folgezuständen der Verletzung.

\section{Letalität}

Die Letalität einer Beckenverletzung wird durch diejenige der Begleitverletzungen wesentlich beeinflusst. So wird für isolierte Beckenfrakturen eine Letalität von unter $2 \%$ [6] genannt, während die Letalität mit Begleitverletzungen für Frakturen des ventralen Beckenrings mit 8,8\% und des dorsalen mit 14\% angegeben werden [2].

Die Letalität wird hierbei v.a. durch das Ausmaß und Dynamik des Blutverlustes bestimmt.

\section{Thrombose- und Embolierisiko}

Die Zahl der beobachteten tiefen Beckenund Beinvenenthrombosen hängt stark von den angewandten diagnostischen Verfahren ab, muss aber als hoch eingeschätzt werden, da lokal eine direkte Endothelschädigung, eine lokale Schwellung oder Hämatome bestehen können und aufgrund der Mehrfachverletzung eine Hyperkoagulabilität auftritt. In manchen Untersuchungen sind bis $\mathrm{zu}$ $61 \%$ tiefe Beinvenenthrombosen und bis 29\% Beckenvenenthrombosen beschrieben [1].

\section{Versorgungsassoziiertes Infektrisiko}

Für die Stabilisierung der Symphyse wird das Infektrisiko mit ca. 3 bis $13 \%$ angegeben.

Bei der Versorgung des Sakroiliakal-Gelenks treten Infekte zwischen ca. 2 und $4 \%$ beim vorderen und zwischen 3,3 und $9,1 \%$ beim hinteren Zugang auf. Infektraten bei der Versorgung mittels Fixateur externe sind noch größeren Schwankungsraten unterworfen (0$33 \%$ ), wobei es sich hier im Wesentlichen um Infekte der Pinaustrittsstellen handelt, Osteomyelitiden oder Sepsis werden sehr selten beobachtet.

\section{Nervenverletzungen}

Am meisten gefährdet und verletzt ist aufgrund der Lagevariabilität und seiner Exposition der N.cutaneus femoris lateralis. Bedeutender ist jedoch die Verletzungsgefahr für den Plexus lumbosacralis, welche bei der Plattenosteosynthese der SIVerletzung droht, aber auch indirekt durch Schraubenfehllage bei transiliosakraler Verschraubung erfolgen kann.

\section{Hernien}

Hernien gelten als seltene Komplikationen. Sie können suprapubisch, inguinal oder lumbal auftreten, fraktur- oder versorgungsbedingt sein. Zotter und Titze konnten bei konservativ therapierten vorderen Beckenringverletzungen 8,8\% Leistenhernien beobachten.

\section{Spätergebnisse}

\section{Schmerz}

Schmerzen sind die häufigsten Probleme nach Beckenfrakturen (12-70\%). Ihre Ausprägung korreliert im Wesentlichen mit der primären Instabilität der Beckenfraktur und der postoperativ verbliebenen Dislokation.

Eine multizentrische Studie der Arbeitsgruppe Becken der Deutschen Sektion der AO International und der Deutschen Gesellschaft für Unfallchirurgie hat ergeben, dass ca. 20-40\% aller Patienten mit B-Verletzungen und 30-37\% nach
C-Verletzungen langfristig unter mittelstarken bis starken Schmerzen leiden.

\section{Neurologische Defizite}

Neurologische Einschränkungen nach instabilen Beckenfrakturen werden in ca. $1 / 5$ der Fälle beobachtet. Hierbei ist hauptsächlich der N. ischiadicus und dieser v.a. durch motorische Ausfälle betroffen. Die Inzidenz korreliert mit dem Grad der primären Instabilität und dem gleichzeitigen Vorliegen einer Komplexverletzung.

\section{Urologische Defizite}

Das Ausmaß bleibender urologischer Schäden korreliert ebenfalls mit dem Vorliegen einer Komplexverletzung. Es werden Miktionsbeschwerden (8,8\%) als auch Einschränkungen der Potenz (13\%) und Sphinkterstörungen (6,6\%) beschrieben. Störungen von Blasenfunktion und Potenz sind besonders häufig bei zentralen Sakrumfrakturen.

\section{Endergebnis}

Für ein zufrieden stellendes Langzeitergebnis ist eine exakte Reposition Voraussetzung. Selbst wenn dies gelingt, sind nicht wenige Patienten dauerhaft durch relevante irreversible Langzeitschäden beeinträchtigt ( $\mathrm{z}$. B. neurologische, urologische Defizite).

\section{Schlussfolgerung}

Ziel bei der Versorgung von instabilen Beckenfrakturen ist eine rasche mechanische Stabilisierung. Obschon eine frühe definitive Versorgung wünschenswert ist, steht ihr meist der labile Kreislaufzustand des Patienten entgegen, so dass überbrückend eine temporäre Stabilisierung notwendig wird.

Die definitive Versorgung ist meist nicht vor dem dritten Tag möglich, sollte aber auch nicht später als 14 Tage nach dem Unfall erfolgen, da durch eine rasche Kallusbildung eine adäquate Reposition und Osteosynthese deutlich erschwert wird.

Dieses Zeitintervall muss für eine gute Vorbereitung der Operation hinsichtlich Diagnostik (CT), Klassifikation (Stabilität) und OP-Planung (Implantate, Instrumente, Durchleuchtung) genutzt werden.

Zur Versorgung sind spezielle Repositionszangen, Rekonstruktionsplatten, 

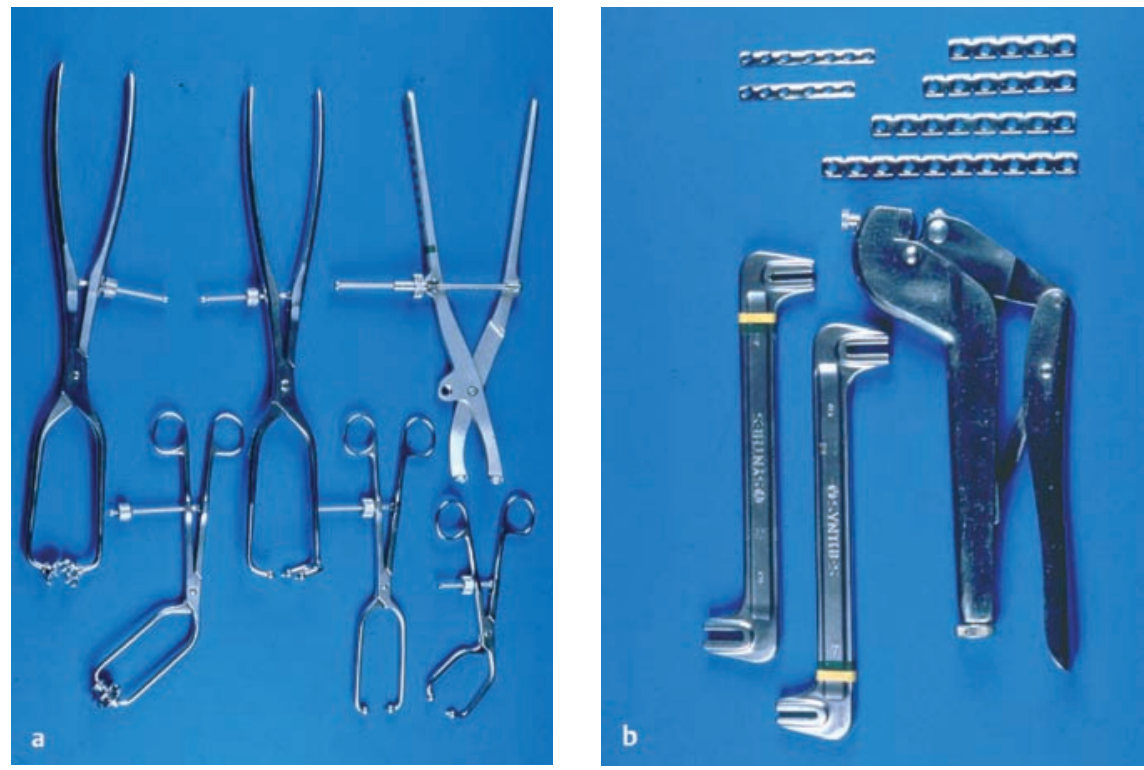

Abb.8au.b Repositionszangen, Rekonstruktionsplatten, Biegepresse und Schränkeisen sind unverzichtbare Werkzeuge in der Beckenchirurgie.

lange Bohrer und Schrauben notwendig. Vor Operationsbeginn muss darauf geachtet werden, dass der Patient optimal gelagert wird und eine Durchleuchtung in den notwendigen Ebenen problemlos möglich ist.

Zur Versorgung von Beckenfrakturen steht eine Reihe von operativen $\mathrm{Zu}-$ gängen zur Verfügung. Die Wahl des Zugangs ergibt sich aus der beabsichtigten
Stabilisierung und muss neben einer ausreichenden Exposition der Fraktur immer gewährleisten, dass der Weichteilschaden oder die Gefahr der Verletzung wesentlicher Strukturen (Nerven, Gefäße, Urogenitaltrakt,...) minimal gehalten wird. Das Erreichen und Halten einer exakten Reposition des knöchernen Beckenringes hat sich als ausschlaggebend für ein gutes Langzeitergebnis herausgestellt.

\section{Literatur}

${ }^{1}$ Geerts W, Coren K, Jay R et al. A prospective study of venous thromboembolism after major trauma. N Engl J Med 1994; 331(24): $1601-1606$

2 Looser K, Crombie H. Pelvic fractures: an anatomic guide to severity of injury. Review of 100 cases. Am I Surg 1976; 132: 638 - 645

3 Parkkari J, Kannus P, Niemi S et al. Secular trends in osteoporotic pelvic fractures in finnland: number and incidence of fractures in 1970 - 1991 and prediction for the future. Calcif Tissue Int 1996; 59(2): 79-83

4 Pohlemann T. In: Tscherne H., T. Pohlemann: Unfallchirurgie - Becken und Acetabulum Springer, Berlin Heidelberg 1998, S. 212

5 Pohlemann T, Gänsslen A. Komplexe Verletzungen des Beckens und Acetabulums. Orthopäde 1998; 27: $32-44$

6 Pohlemann T, Gänsslen A, Kiessling B et al. Indikationsstellung und Osteosynthesetechniken am Beckenring. Unfallchirurg 1992; 95: 197-209

7 Schmal H, Klemt C, Haag C et al. Die komplexe Beckenverletzung im Kindesalter. Unfallchirurg 2002; 105: 748-754

8 Young JW, Burgess AR, Brumback RJ et al. Pelvic fractures: value of plain radiography in early assessment and management. Radiology 1986; 160(2): 445-451

\section{Dr. med. Jochen Pressmar} Assistenzarzt

\section{Prof. Dr. med. Lothar Kinzl}

Chefarzt

Dr. med. Michael Robindra Sarkar Unfallchirurg

Abteilung für Unfall-, Hand- und Wiederherstellungschirurgie Steinhövelstr. 9 D-89075 Ulm 